\title{
ALTIMETRY STUDY PERFORMED USING AN AIRBORNE GNSS-REFLECTOMETER
}

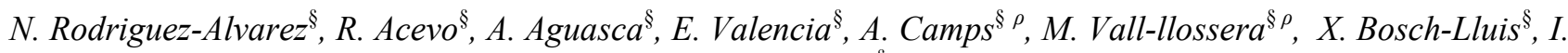 \\ Ramos-Perez ${ }^{\S}$
}
${ }^{\S}$ Remote Sensing Lab, Dept.Teoria del Senyal i Comunicacions, Building D3, Universitat Politècnica de Catalunya and IEEC CRAE/UPC, 08034 Barcelona, Spain.
${ }^{\rho}$ SMOS Barcelona Expert Centre. Pg. Marítim de la Barceloneta 37-49, 08003 Barcelona, Spain

Tel.+34+934017362, E-mail: nereida@tsc.upc.edu

\begin{abstract}
The Global Navigation Satellite Signals Reflections (GNSS$\mathrm{R})$ techniques have been widely used for remote sensing purposes retrieving geophysical parameters over different types of surfaces. Over the ocean, altimetry $[1,2]$ or sea state $[3,4]$ can be retrieved. Over land, soil moisture $[5,6]$ can be inferred and over ice, altimetry, and ice age [7] are also retrieved. This paper presents the results of using GNSS-R techniques to retrieve altimetry from the measurements of an airborne GNSS-Reflectometer.
\end{abstract}

Index Terms - GNSS-R, Airborne reflectometer, Altimetry

\section{INTRODUCTION}

A lot of work has been carried out in altimetry applications of GNSS-R [1,2]. This paper presents another contribution to the altimetry retrieval using GNSS-R techniques but from a small remote control aircraft. The first part of this work focuses on the developed reflectometer, its main structure and characteristics. The second part of this paper focuses on a set of measurements performed with synthetic data generated with a vector signal generator in order to validate the system. The third part of the paper shows the experimental field campaign and the main results achieved. Finally, the conclusions are presented.

\section{GNSS-REFLECTOMETER}

The GNSS-Reflectometer is mainly composed of two antennas, one antenna for the direct signal and one antenna for the reflected signal. These two antenna signals are combined, amplified, downconverted and sampled at 8.18 MHz. The GNSS-Reflectometer is a small instrument suitable for airborne applications, such as a radio control aircraft (Fig. 1) developed and controlled by the UPC's professor Albert Aguasca.

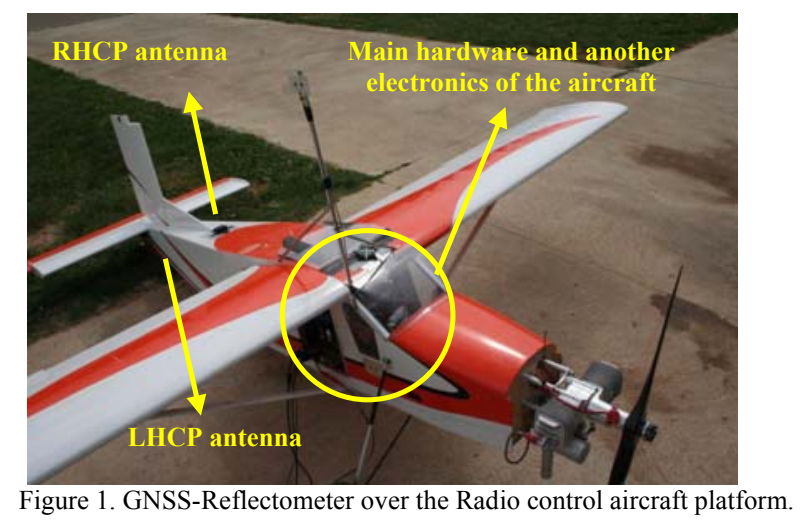

The raw GPS data is collected using an USB interface and stored into a binary file in the on-board computer.

Then, applying GNSS-R signal processing techniques, the measured direct and reflected signals are simultaneously correlated with a local replica of the PRN code, to compute the Delay-Doppler Map (DDM) [8]. This DDM has two peaks (one is due to the direct signal and the other to the reflected signal). In order to visualize them, the radio control aircraft must fly high enough to separate in delay samples the reflected signal from the direct signal, avoiding that the direct signal masks the less intense reflected signal.

\section{THEORETICAL SIMULATIONS}

The expected product given by the GNSS-Reflectometer is two DDM peaks coming from the same satellite, but one delayed with respect to the other, due to the differences in the paths covered, and one has a smaller amplitude than the other, due to the reflectivity of the surface over which the GPS signal is scattering. In order to emulate these measurements a vector signal generator has been used, SMU 200A Vector Signal Generator from ROHDE SCHWARZ [9]. 


\subsection{Signal levels and incoherent integration time}

The level of the two signals is different due to the fact that the reflected signal comes from the surface over which has been scattered and affected by their reflectivity. So, that the reflected signals is expected to be powerless than the direct signal.

In order to consider the received signals as quality enough signals, the minimum SNR that must be reached is 5, following the Rose criterion [10] (eqn.1).

$$
S N R=\frac{\text { Signal }_{\text {peak }}-\mu_{\text {noise }}}{\sigma_{\text {signal }}}
$$

The weaker the reflected signals, the larger the incoherent integration time needed to achieve a SNR $>5$ is. In fig. 2 a number of measurements with synthetic data has been performed considering the reflected signal some $\mathrm{dB}$ lower than the direct one and the corresponding SNRs has been computed by increasing the incoherent integration time.

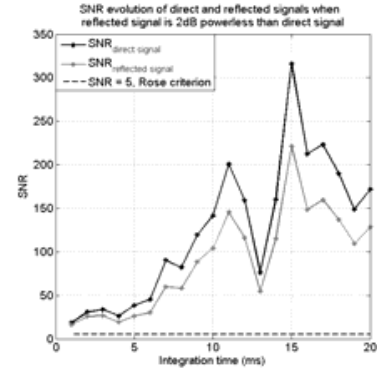

(a)

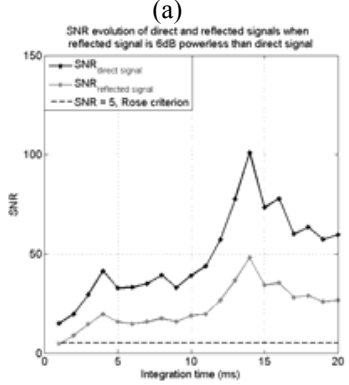

(c)

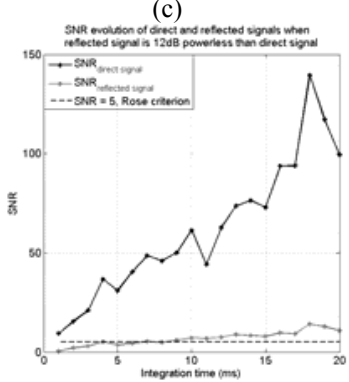

(e)

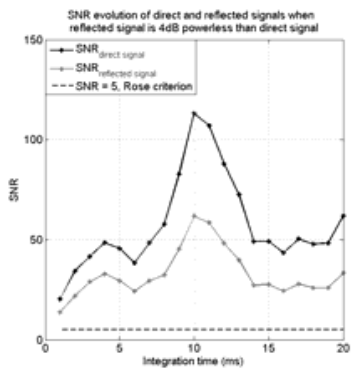

(b)

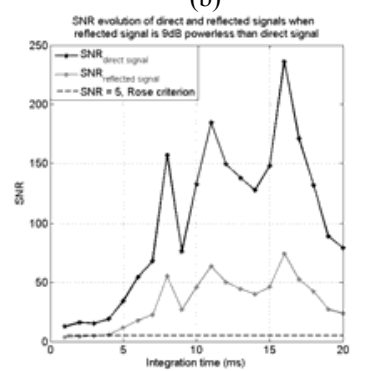

(d)

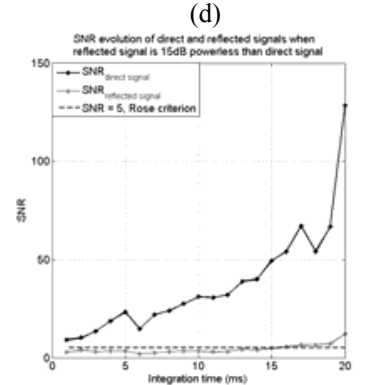

(f)
Figure 2. Simulated direct signal SNR and especular reflection signal SNR considering: reflected signal (a) $2 \mathrm{~dB}$, (b) $4 \mathrm{~dB}$, (c) $6 \mathrm{~dB}$, (d) $9 \mathrm{~dB}$, (e) $12 \mathrm{~dB}$ and (f) $15 \mathrm{~dB}$ lower than direct signal
As it can be seen when reflected signal is attenuated 2 $\mathrm{dB}$ respect to the direct one the required coherence integration time is $1 \mathrm{~ms}$, but in the extreme case where reflected signal is $15 \mathrm{~dB}$ lower than the direct one, $16 \mathrm{~ms}$ incoherence integration is needed.

\subsection{Instrument height and DDMs separation}

Depending on the aircraft altitude and the surface topography the distance covered by the reflected signal can be greater or smaller. The path difference $(\Delta y)$ between the direct and the reflected signals translates in a delay difference ( $N_{\text {samples }}$ ) in the DDM obtained, eqn.2.

$$
\Delta y=\frac{c}{f_{s}} \cdot N_{\text {samples }}=\frac{c}{f_{s}} \cdot \frac{N_{\text {chips }}}{(1 \mathrm{~ms} / 1023) \cdot f_{s}}
$$

where $c$ is the light speed and $f_{s}$ is the sampling frequency of the GNSS-Reflectometer, and $N_{\text {chips }}$ is the number of chips in one period. Figure 3 shows the simulation of the two peaks (direct and reflected) splitting as a function of the number of chips introduced.

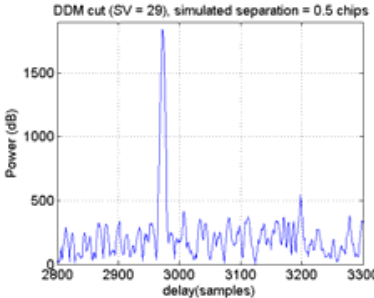

(a)

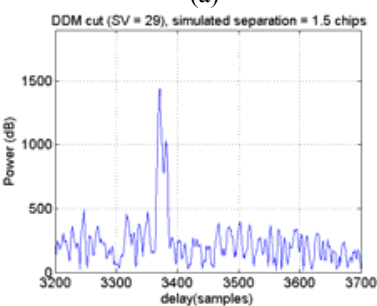

(c)

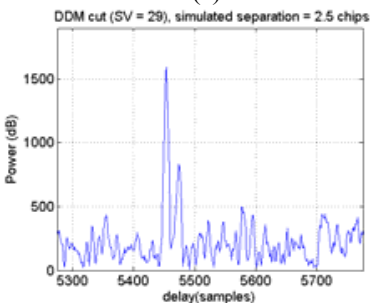

(e)

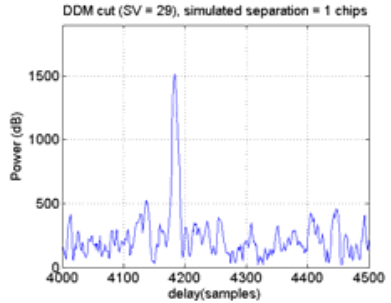

(b)

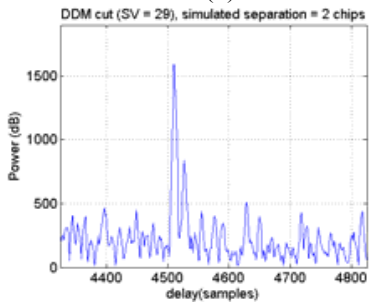

(d)

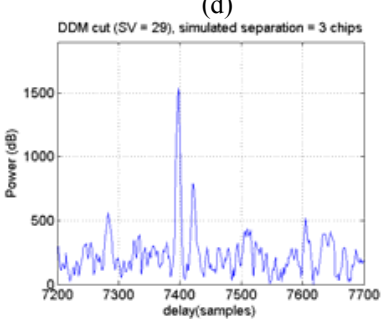

(f)
Figure 3. . Simulated direct signal and especular reflection signal by introducing a delay in the reflected signal of (a) 0.5 chips, (b) 1 chip, (c) 1.5 chips, (d) 2 chips, (e) 2.5 chips and (f) 3 chips.

As it can be seen, reflected signal must be at least 2 chips splitted to be able to avoid overlapping between direct 
and reflected signals. Since $f_{s}=8183.86 \mathrm{MHz}$ and computing eqn. 2, a path difference of $\Delta y=582.52 \mathrm{~m}$ is achived, which is equivalent to a flight height of $h_{\text {flight }}=\frac{\Delta y}{2}=293.26 \mathrm{~m}$. Therefore the minimum flight height required is $293.26 \mathrm{~m}$.

The resolution of the system is 1 sample, which is equivalent to $18.33 \mathrm{~m}$ height. This resolution obtained from the delay of the DDM peaks could be improved using the phase of the DDM peaks.

\section{EXPERIMENTAL RESULTS}

\subsection{Field experiment setup}

In order to acquire some raw data a field campaign was performed over an airfield at Ripollet, Spain, on June $29^{\text {th }}$, 2009. Figure 4 shows the aircraft trajectory (blue dots) and the GNSS-Reflectometer successful measurements in which both peaks are present (named as "shots" and marked in yellow colour).

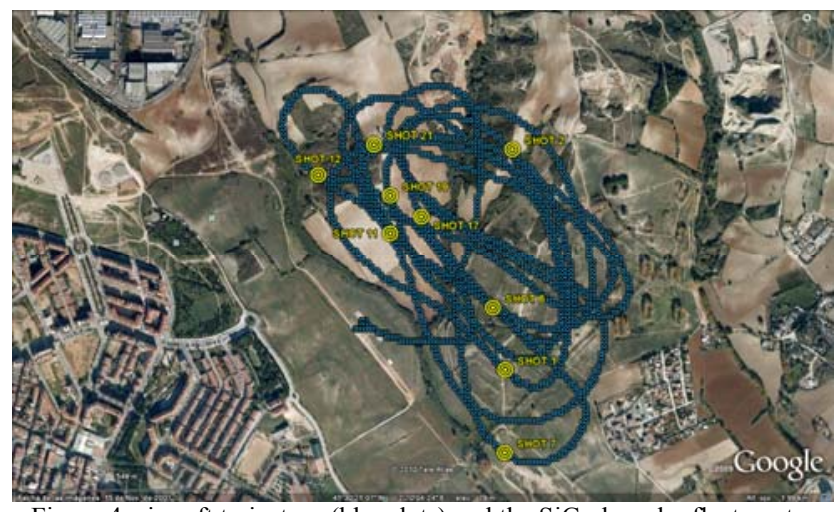

Figure 4. aircraft trajectory (blue dots) and the SiGe-based reflectometer successfully shots (yellow dots)

The main data for the aircraft attitude and altitude has been acquired using X-sens MTi-G sensor [11]. Attitude (roll and pitch angles) are shown in Fig. 5, and also an image of the aircraft during the flight.

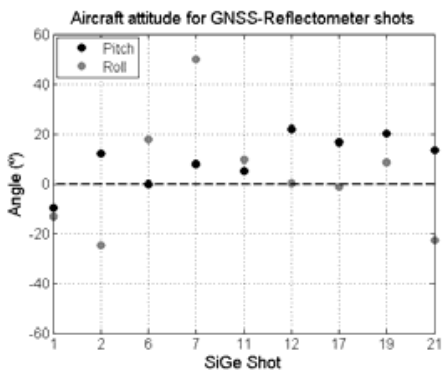

(a)

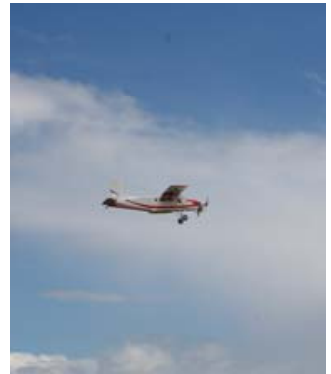

(b)
Figure 5. (a) Attitude (roll and pitch angles), (b) aircraft image in flight

\subsection{Results}

Some of the successful shots are shown in Fig. 6.

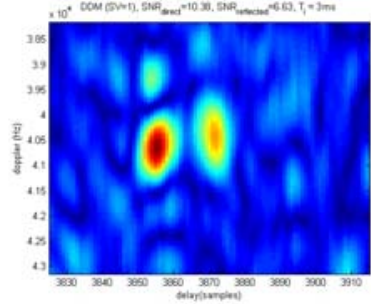

(a)

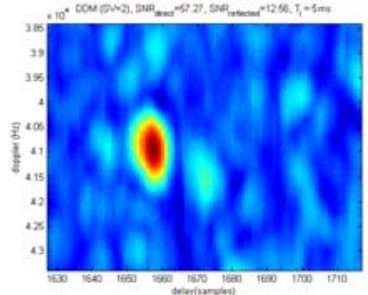

(c)

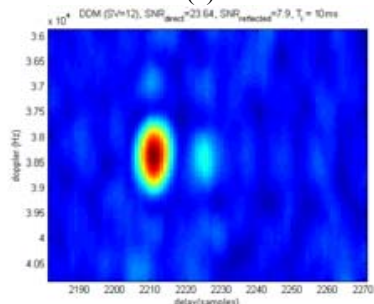

(e)

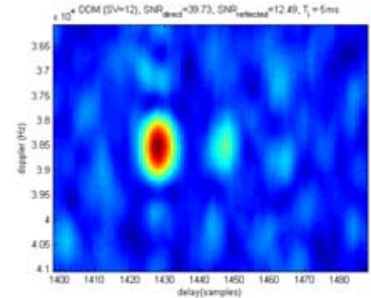

(b)

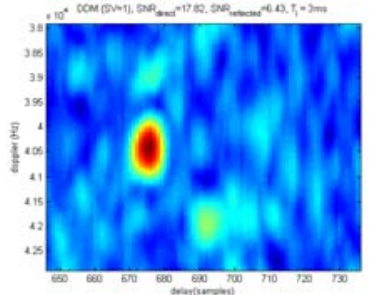

(d)

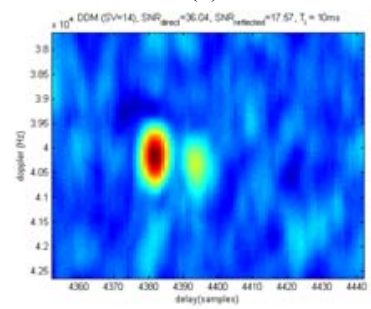

(f)
Figure 6. Two peaks DDMs obtained in shot number (a) 1, (b) 6, (c) 7, (d) 12, (e) 17 and (f) 19 .

Each one of the measurements has required a different incoherent integration time in order to achieve a quality enough SNR (see Fig. 6 plot headers). The number of samples between the direct and reflected signals has been obtained and the equivalent flight height has been computed using the rationale explained in section 3.2, Fig.7. The differences are due to the following reasons:

- X-sens gives altitude in WGS-84 (respect to the geoide) while GNSS-Reflectometer gives the topographical distance (respect to the ground surface).

- X-sens gives the altitude respect to the gravity vector while GNSS-Reflectometer gives the distance to the point where signal is reflected. It means that, due to the elevation and azimuth angles of the satellites, the reflectometer measurement does not have to match the $\mathrm{X}$-sens data point. This difference can be corrected by knowing the satellites position, but this information was not available in the field experiment.

- Pitch and roll angles must be as close to zero as possible to avoid undesirable antenna effects. 


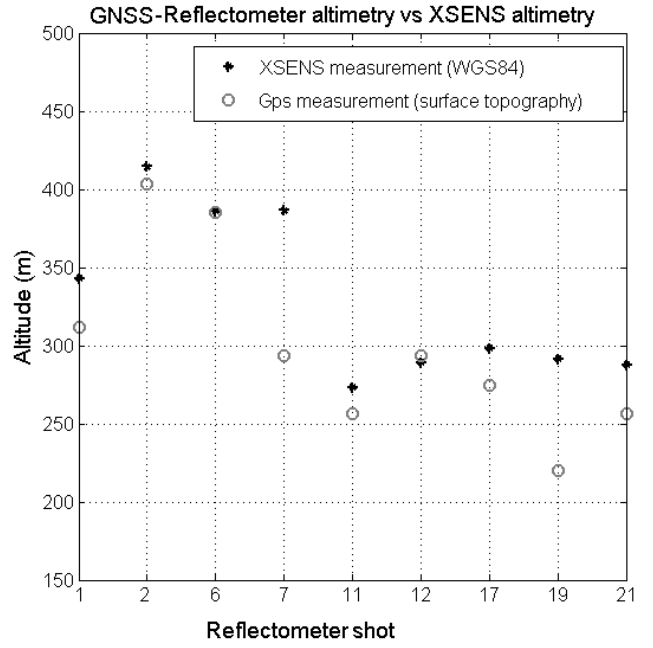

Figure 7. X-sens ground truth vs GNSS-Reflectometer retrieved heights.

\section{CONCLUSIONS}

The main conclusions extracted from theoretical simulations have been summarized using Fig. 8 .

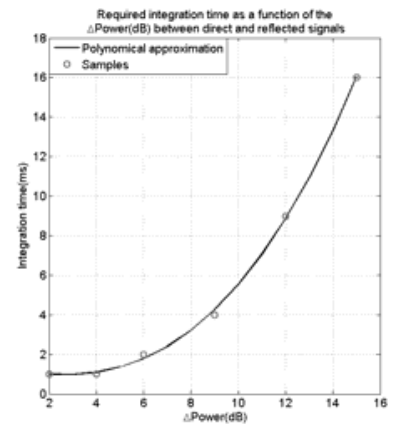

(a)

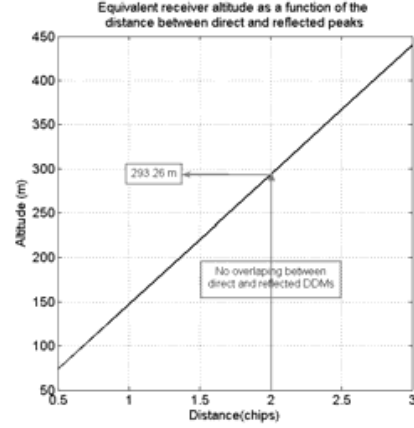

(b)
Figure 8. Main conclusions obtained: (a) Required integration time as a function of the power difference between direct and reflected signals, and (b) equivalent receiver altitude as a function of the distance between direct and reflected peaks.

Figure $8 \mathrm{a}$ shows the relationship between the power difference and the incoherence integration time needed to obtain quality DDMs. This relationship follows a third order polynomial approximation. Figure $8 \mathrm{~b}$ shows the linear relationship between altitude as a function of the distance between direct and reflected peaks.

From the experimental results it is concluded that topography retrieval can be achieved using the GNSSReflectometer, but some improvements must be done to the experimental setup in order to give accurate results: as knowing the topography, to validate the retrieval, and having information about satellite elevation and azimuth, to better assign the retrieved heights to surface location.

\section{ACKNOWLEDGEMENT}

This work was supported by funds from the Plan Nacional del Espacio of the Spanish Ministry in the frame of the project with reference ESP2007-65567-C04-02. And also by funds from the project with reference AYA2008-05906-C02-01/ESP

\section{REFERENCES}

[1] Martín-Neira, M., "A Passive Reflectometry and Interferometry System (PARIS): Application to Ocean Altimetry." ESA Journal, vol. 17. pp 331-355, 1993.

[2] Martin-Neira, M., P. Colmenarejo, G. Ruffini, C. Serra, "Ocean Altimetry using the Carrier Phase of GNSS Reflected Signals," Proceedings of Ocean Winds 2000, Plouzane, France, CERSAT News, Issue 11, November 2000.

[3] Soulat, F., M. Caparrini, O. Germain, P. Lopez-Dekker, M. Taani, G. Ruffini, "Sea state monitoring using coastal GNSSR," Geophysical Research Letters, vol. 31, no. 21.

[4] Marchan-Hernandez, J.F., N. Rodríguez-Álvarez, A. Camps, X. Bosch-Lluis, I. Ramos-Perez, E. Valencia, "Correction of the Sea State Impact in the L-band Brightness Temperature by Means of Delay-Doppler Maps of Global Navigation Satellite Signals Reflected over the Sea Surface," IEEE Transactions on Geosceince and Remote Sensing, October 2008.

[5] Masters, D., V. Zavorotny, S. Katzberg and W. Emery, "GPS signal scattering from land for moisture content determination," Proceedings of the Internacional Geoscience and Remote Sensing Symposium 2000, vol.7 pp. 3090-3092, Honolulu, USA.

[6] Rodriguez-Alvarez, N., Bosch-Lluis, X., Camps, A., Vall-llossera, M., Valencia, E., Marchan-Hernandez, J.F., Ramos-Perez, I.; "Soil moisture retrieval using GNSS-R techniques: experimental results over a bare soil field," IEEE Transactions on Geoscience and Remote Sensing, Vol. 47 (11), pp. 245-248, November 2009.

[7] Komjathy, A., J. Maslanik, V. Zavorotny, P. Axelrad and S. Katzberg, "Sea Ice Remote Sensing Using Surface Reflected GPS Signals," Proceedings of the Internacional Geoscience and Remote Sensing Symposium 2000, Honolulu, USA.

[8] Zavorotny, V. U., and A.G. Voronovich, "Scattering of GPS Signals from the Ocean with Wind Remote Sensing Application," IEEE Transactions on Geoscience and Remote Sensing, vol. 35, no. 3, pp. 951-964, March 2000.

[9] SMU 200A Vector Signal Generator from ROHDE SCHWARZ

http://www.rohde-schwarz.es/product/SMU200A.html [10] A. Rose "Vision human and electronic," Plenum Press, 1973

[11] X-sens MTi-G sensor

http://www.Xsens.com/en/general/mti-g 